\title{
Fluid-Structure Interaction Study on Diffuser Pump With a Two-Way Coupling Method
}

\author{
Xu Huan, Liu Houlin, Tan Minggao and Cui Jianbao \\ Research Center of Fluid Machinery Engineering and Technology, \\ Jiangsu University, Zhenjiang, Jiangsu 212013, China, xhqq_1234@126.com
}

\begin{abstract}
In order to study the effect of the fluid-structure interaction (FSI) on the simulation results, the external characteristics and internal flow features of a diffuser pump were analyzed with a two-way flow solid coupling method. And the static and dynamic structure analysis of the blade was also caculated with the FEA method. The steady flow field is based on Reynolds Averaged N-S equations with standard $k-\varepsilon$ turbulent model, the unsteady flow field is based on the large eddy simulation, and the structure response is based on elastic transient structural dynamic equation. The results showed that the effect of FSI on the head prediction based on CFD really exists. At the same radius, the van mises stress on the nodes closed shroud and hub was larger than other nodes. A large deformation region existed near inlet side at the middle of blades. The strength of impeller satisfied the strength requirement with static stress analysis based on the fourth strength theory. The dynamic stress varied periodically with the impeller rotating. It was also found that the fundamental frequency of the dynamic stress is the rotating frequency and its harmonic frequency. The frequency of maximum stress amplitude at node 1626 was 7 times of the rotating frequency. The frequency of maximum stress amplitude at node 2328 was 14 times of the rotating frequency. No matter strength failure or fatigue failure, the root of blades near shroud is the key region to analyse.
\end{abstract}

Keywords: Fluid-Structure Interaction, Diffuser Pump, Two-Way Coupling Method, head prediction

\section{Introduction}

The existing research for unsteady flow field and the corresponding flow induced vibration analysis of centrifugal pump are mainly carried out respectively without considering the interaction between fluid and structure. The ignorance of fluid structure interaction (FSI) means that the energy transfer between fluid and structure is neglected. To some extent, the accuracy and reliability of unsteady flow and rotor deflection analysis should be affected by this interaction mechanism.

FSI is a subset of multi-physics applications. Following different methods for numerical solutions can be used to solve FSI problem:

(1) One-way coupling: This practical approach treats the fluid physics and the solid physics consecutively. Only the flow affects the solid, no feedback from the solid to the flow is considered.

(2) Two-way coupling: For stronger coupled physics the deformations and/or displacements of the solid have a more or less significant impact on the flow. For this case a feedback from the solid analysis to the flow solution is required to achieve meaningful results.

(3) Direct coupling: In cases where the flow and the solid physics are inseparable linked together, the governing equations of the physics of the fluid and the solid must be solved simultaneously. This approach seems to be ideal when the physical interactions are strongly non-linear. At present this method is practicable only for elementary examples. For most technical devices a simultaneous numerical solution of the governing equations is hard to achieve because of the complexity of the geometry and the therefore required large computer resources.

In recent years, more and more applications of FSI in the reliability research of turbomachinery. Most of them are about turbine [13], and a few of them are pump. Kato [4] predicted the noise from a multi-stage centrifugal pump using one-way coupling method. Benra[5]discussed the flow-induced vibrations of a commercial single-blade sewage water pump in detail with numerical and experimental methods, and indicated that comparing with one-way coupling method, two-way coupling method got a significant result. Yang Wang [6] caculated the stress and deformation of the impeller for a stamping and welding centrifugal pump in ANSYS with Oneway coupling method. Ji Pei [7] used two-way coupling method to study the effect of FSI of impeller on flow field in centrifugal pump.

Received July 6 2012; revised January 18 2013; accepted for publication February 28 2013: Review conducted by Prof. Hyung-Hee Cho. (Paper number O13003S)

Corresponding author: Xu Huan, Master, xhqq_1234@126.com 
In this paper, CFD solver ANSYS-CFX and FEM solver ANSYS were applied to calculate the flow and structure field for a diffuser pump based on a two-way coupling method. The purpose of this research was to learn about the effect of the FSI on prediction of the external characteristics. Because there is no report that FSI is applied to the prediction of external characteristics. Then the static structure analysis of the blade was caculated. Finally, the dynamic stress was discussed. Because there is also no report that two-way coupling method is used on strength calculation of pumps.

\section{Pump Model}

The model is a residual heat removal pump. The designed flow rate is $900 \mathrm{~m}^{3} / \mathrm{h}$, with head of $79 \mathrm{~m}$ and rotating speed of 1490 $\mathrm{r} / \mathrm{min}$. The impeller is enclosed type with 5 blades. In order to improve flow condition and balance radial force, the guide vanes are installed between impeller and annular chamber. The number of guide vane is 7 for the protection of the resonance.

\section{FSI simulations with a two-way coupling}

\subsection{Numerical simulation of flow field}

The water field is meshed with the unstructured grid by the ICEM CFD codes (see Fig. 1(a)).The number of the grid element is 3166576.The internal flow of diffuser pump is analysed by CFD code ANSYS-CFX12.0. The boundary conditions are as follow.

(1) Inlet: total pressure, $1 \mathrm{~atm}$.

(2) Outlet: normal speed, $5.15 \mathrm{~m} / \mathrm{s}$.

(3) Wall: general boundary condition by default.

The turbulence model of steady simulation used standard $k-\varepsilon$ model, scalable wall function. The simulation result of the transient internal flow based on LES method is better than the RANS method [8], so the LES method is used in the unsteady simulation. The Smagorinsky turbulence model is used as well as the automatic wall function.

The Smagorinsky model is an algebraic model for the SGS viscosity $\boldsymbol{v}_{\mathrm{SGS}}$. Based on dimensional analysis, the SGS viscosity can be expressed as:

$$
v_{S G S} \propto l q_{S G S}
$$

Where $l$ is the length scale of the unresolved motion (usually the grid size $\Delta=(\mathrm{Vol}){ }^{1 / 3}$ ) and $q_{\mathrm{SGS}}$ is the velocity of the unresolved motion.

Based on an analogy to the Prandtl mixing length model, the velocity scale is related to the gradients of the filtered velocity:

$$
\begin{gathered}
q_{S G S}=\Delta|\bar{S}| \\
|\bar{S}|=\left(2 \overline{S_{i j}} \overline{S_{i j}}\right)^{1 / 2}
\end{gathered}
$$

This yields the Smagorinsky model for the subgrid-scale (SGS) viscosity:

$$
v_{S G S}=\left(C_{S} \Delta\right)^{2}|\bar{S}|
$$

with $C_{S}$ the Smagorinsky constant. The value of the Smagorinsky constant for isotropic turbulence with inertial range spectrum:

$$
\begin{gathered}
E(k)=C_{k} \varepsilon^{2 / 3} k^{-5 / 3} \\
C_{S}=\frac{1}{\pi}\left(\frac{2}{3 C_{k}}\right)^{3 / 4}=0.18
\end{gathered}
$$

For practical calculations, the value of $C_{S}$ is changed depending on the type of flow and mesh resolution. Its value is found to vary between a value of 0.065 (channel flows) and 0.25 . Often a value of 0.1 is used and has been found to yield the best results for a wide range of flows. This is also the default value of $C_{S}$.

The coefficient $C_{\mathrm{S}}$ is, therefore, not a universal constant and this is the most serious shortcoming of this model. Furthermore, damping functions are needed close to walls.

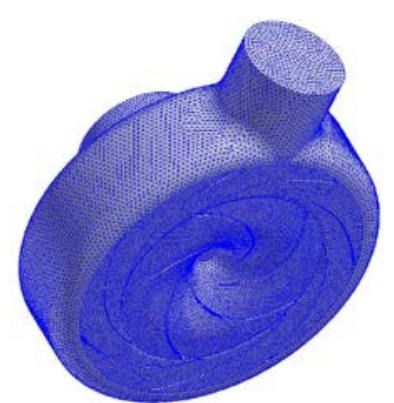

(a) fluid field

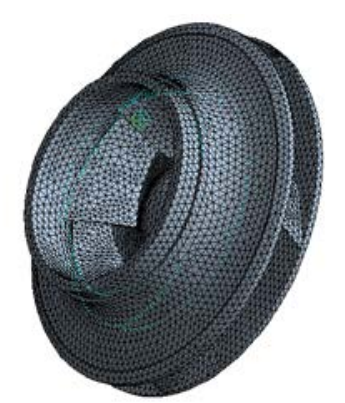

(b)solid field

Fig. 1 Numerical model of fluid and structure

Every 6 degree the impeller rotated is defined as one time step, so time step is $0.000671 \mathrm{~s}$ and total time is $0.24156 \mathrm{~s}$ (6 cycles of impeller). Impeller-volute interface uses general connection interface model, transient rotor stator frame change/mixing model and GGI mesh connection method. The blade surface and the inner face of hub and shroud are set to the fluid solid interface. The 
process of a two-way coupling is illustrated in figure 2. For every time step, the total force of the elements of FSI surface is transferred from the CFD solver ANSYS-CFX to the FEM solver ANSYS-Transient Structural. Then, the total mesh displacement of the elements of FSI surface caused by the total force is transferred from the FEM solver ANSYS-Transient Structural back to the CFD solver ANSYS-CFX.

\subsection{Numerical simulation of solid field}

The solid field is meshed by the DS model of the ANSYS Workbench (see Fig. 1(b)).The number of the grid element is 74528. The material of impeller is stainless steel, the density is $7770 \mathrm{~kg} / \mathrm{m}^{3}$, the stress of yield limit $\sigma_{s}$ is $345 \mathrm{MPa}$, the Young's Modulus is 216GPa and Poisson's Ratio is 0.28. The time step and total time of FEM solver is the same as CFD solver. Considering the large computer resources FEM solver required and the less grid number of solid field, the time step can't be too small. Meanwhile, the grid number of fluid field is very large, so the time step can't be too large. So the time step is $0.000671 \mathrm{~s}$ and total time is $0.24156 \mathrm{~s}$ in the CFD solver.

The transient structural analysis of the pump impeller is carried out with ANSYS code. The transient dynamic equation is a linear structure as follows:

$$
M u^{\prime \prime}+C u^{\prime}+K u=F^{S}+F^{F S I}
$$

Where $M$ is structural mass matrix, $C$ is structural damping matrix, $K$ is structural stiffness matrix, $u^{\prime \prime}$ is nodal acceleration vector, $u^{\prime}$ is nodal velocity vector, $\mathrm{u}$ is nodal displacement vector, $F^{S}$ is applied load vector except FSI force, $F^{F S I}$ is the FSI force vector. Newmark time integration method is employed for the solution of the linear Equation.

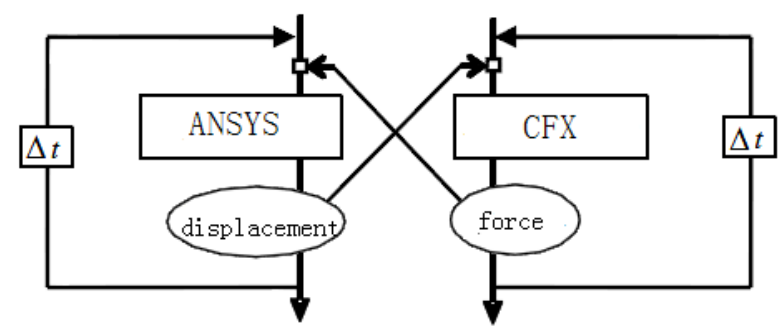

Fig. 2 Two-way flow solid coupling

\section{Results and discussions}

\subsection{Analysis of the external characteristics}

Convergence Residual Target of both numerical solvers is $10^{-4}$. Figure 3 showed the comparison of total head with and without FSI during the last period. The variation tendency of total head was similar during the last period of impeller. But the average of total head with FSI was $84.30 \mathrm{~m}$, and the average of total head without FSI was only $83.96 \mathrm{~m}$ which is lower than the total head with FSI. That means the effect of FSI on the head prediction based on CFD really exists. Therefore, the effect of FSI should be taken into account when the external characteristic of pump is predicated by numerical simulation.

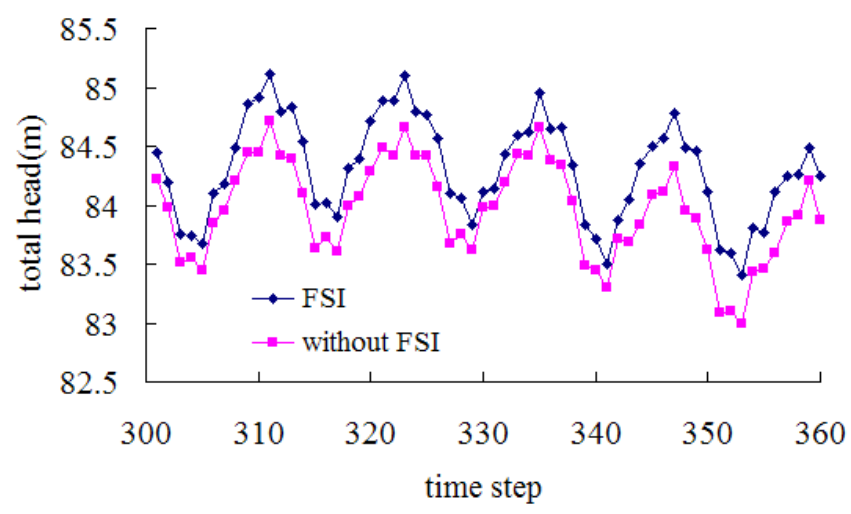

Fig. 3 Total Head

\subsection{Analysis of the pressure distribution on blades}

The results of the last step were analysed. Figure 4 showed the distribution of total pressure on the surface of blades with and without FSI. The variation tendency of total pressure on the surface of blades was similar that increased gradually from inlet to out let. And the total pressure of pressure side was larger than suction side at the same radius. 


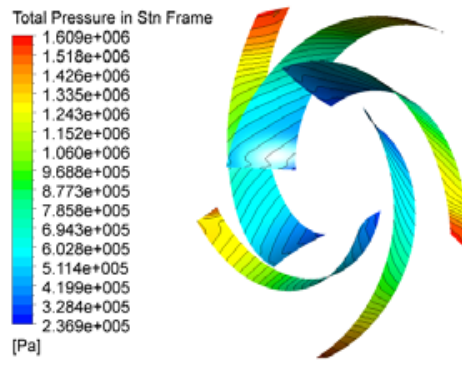

(1) FSI

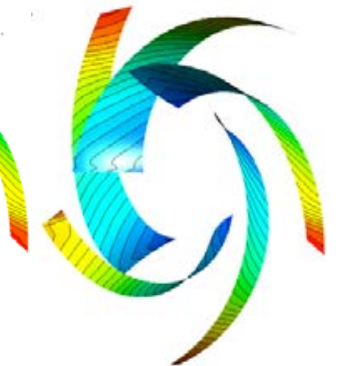

(2) without FSI

(a) pressure side

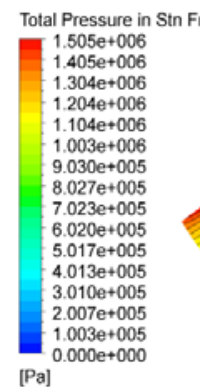

(1) FSI

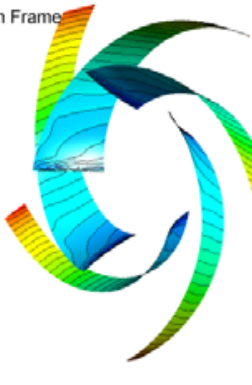

(b) suction side

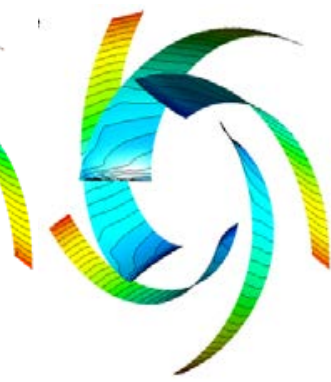

(2) without FSI

Fig. 4 the distribution of total pressure

\subsection{Static analysis of blades stress and deformation}

Figure 5 showed the distribution of von mises stress on the surface of blades. At the same radius, the van mises stress on the nodes closed shroud and hub was larger than other nodes. Because these places exist stress concentration induced by rotating inertial load. The van mises stress on the inlet side of blades is also large, caused by the fluid impact. The maximum von mises stress is on the node 1626 located at the junction of shroud, suction side of blade and outlet of impeller. The total pressure of node 1626 at a sharp point of the blade was largest and stress concentration happened at the sharp point. This region was the common destruction of impeller. The admissible stress $[\sigma]$ of stainless steel:

$$
[\sigma]=\frac{\sigma_{s}}{n_{s}}
$$

Stainless steel is plastic material, $n_{\mathrm{s}}$ takes 1.2 2.0. The maximum von mises stress $\sigma_{e}=123.4 \mathrm{MPa}$, satisfied the strength condition:

$$
\sigma_{e}<[\sigma]
$$

So the impeller satisfied strength requirement.

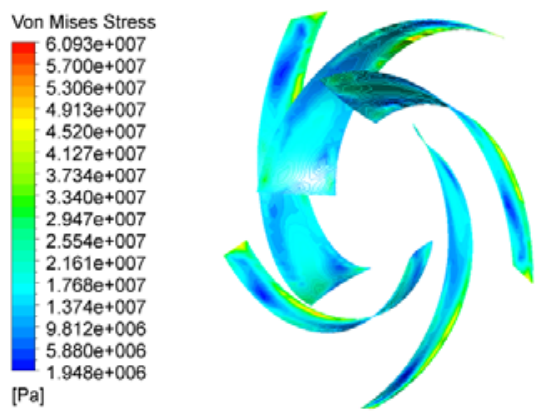

(a) pressure side

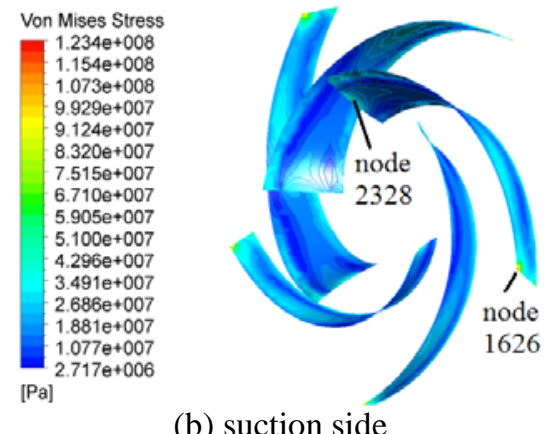

Fig. 5 the distribution of von mises stress 
Figure 6 showed the total mesh displacement of blade from inlet to outlet. In the Figure, the span defines the dimensionless distance (between 0 and 1 ) from the hub to the shroud, span=z/b. Span=0 was defined as the intersection line of blade and hub, span=1 was defined as the intersection line of blade and shroud. Streamline 0-1 defines the dimensionless distance (between 0 and 1) from the inlet to outlet of blades. The mesh displacement variation of pressure side and suction side on blade was similarly. The mesh displacement of span=0.01 and span=0.99 was monotone increasing from inlet to outlet. The mesh displacement of span=0.5 first increased, then decreased. And at the inlet side, the mesh displacement of span=0.99 was larger than span=0.01, and the mesh displacement of span=0.5 was largest. The above difference between span=0.01, span=0.99 and span=0.5 was caused by follow reasons:

(1) The deformation was decided by the stress and the shape of blade, so the mesh displacement variation generally got a good agreement with the fluid load and rotational inertial load.

(2) At the middle stream of blades (span=0.5), the stiffness was the lowest, especially at the inlet of blades.

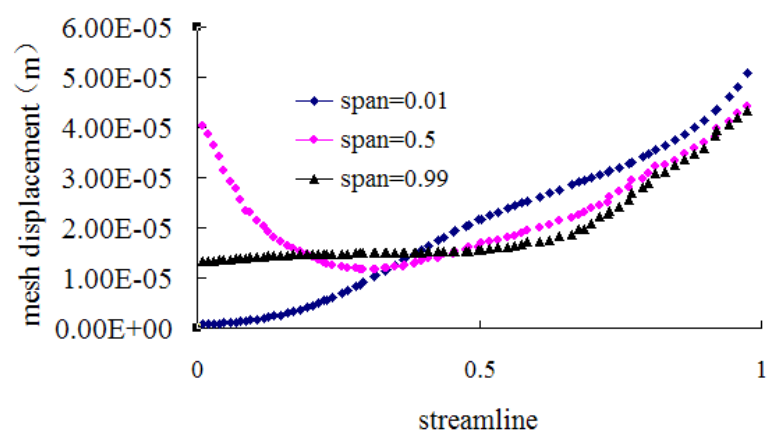

(a) pressure side

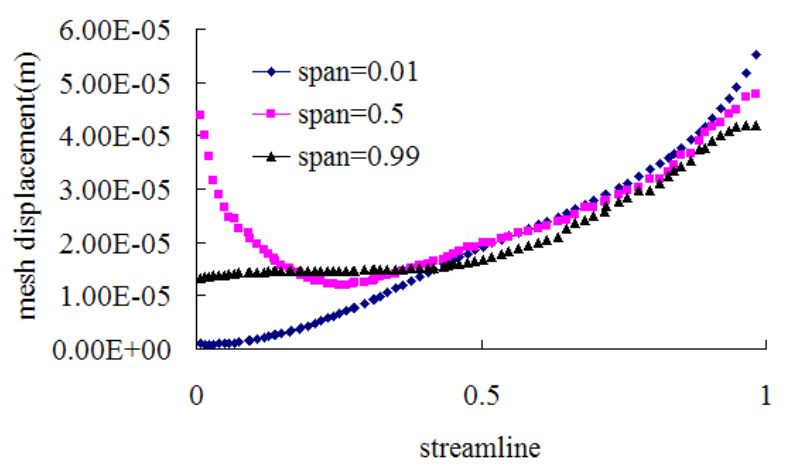

(b) suction side

Fig. 6 total mesh displacement of blade

\subsection{Analysis of dynamic stress}

The fluid pressure pulsation caused by rotor-stator interaction between impeller and guide vane resulted in the dynamic stress and deformation. The dynamic stress would cause the fatigue failure differed from static structure analysis above. So the dynamic stress and deformation of node 1626 and node 2328 were discussed as follow. Node 2328 was a node of the large deformation region at inlet side of blade.

Figure 7 showed the time-domain spectrums of dynamic stress of node 1626 and 2328. During the last period of impeller, the dynamic stress had seven peaks. The number of the peak was the same as the blades of guide vane instead of impeller.

The maximum stress amplitude of node 1626 was larger than node 2328, as shown in figure 8. So when considering the fatigue failure, node 1626 was also the key point. The frequency of maximum stress amplitude at node 1626 was 7 times of the rotating frequency. The frequency of maximum stress amplitude at node 2328 was 14 times of the rotating frequency.

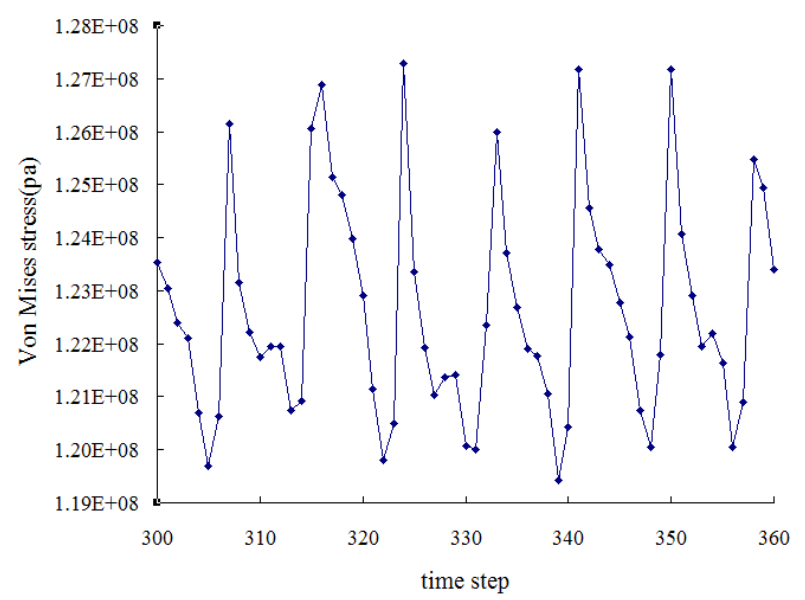

(a) node 1626 


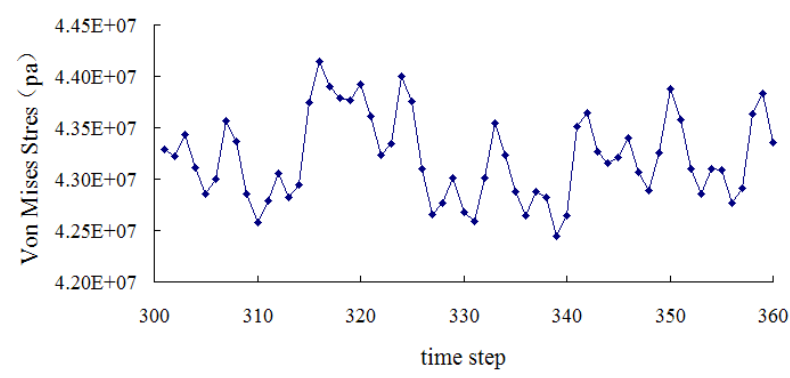

(b) node 2328

Fig. 7 time-domain spectrums of dynamic stress

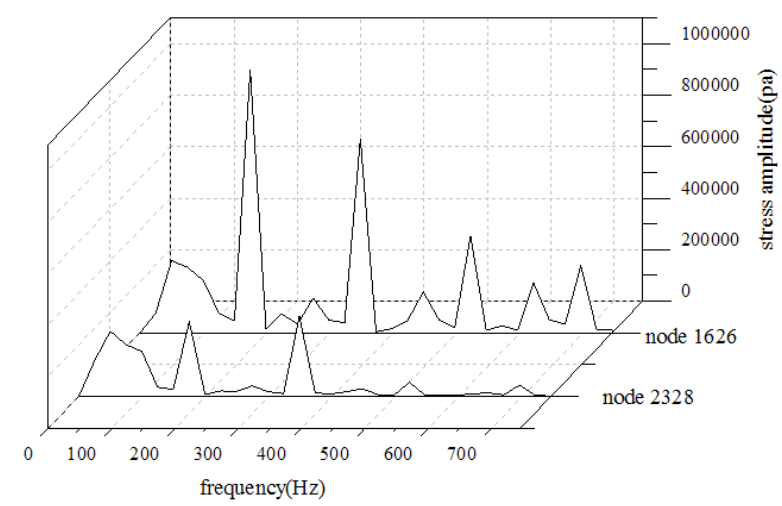

Fig. 8 frequency spectra of dynamic stress

\section{Conclusion}

The external characteristics and internal flow features of a diffuser pump were analyzed with the two-way flow solid coupling method. And the static and dynamic analysis of the blade was also analyzed with the FEA method.

(1) The average of total head with FSI was larger than the total head without FSI. Therefore, the effect of FSI should be taken into account when the external characteristic of pump was predicated by numerical simulation.

(2) At the same radius, the van mises stress on the nodes closed shroud and hub was larger than other nodes. A large deformation region existed near inlet side at the middle of blades. The strength of impeller satisfied the strength requirement with static stress analysis based on the fourth strength theory.

(3) No matter strength failure or fatigue failure, the root of blades near shroud was the key region.

(4) The dynamic stress varied periodically with the impeller rotating. It was also found that the fundamental frequency of the dynamic stress is the rotating frequency and its harmonic frequency. The frequency of maximum stress amplitude at node 1626 was 7 times of the rotating frequency. The frequency of maximum stress amplitude at node 2328 was 14 times of the rotating frequency.

\section{Acknowledgments}

A The authors would like to thank the support by the National Natural Science Foundation of China (Grant No. 50825902, 51079062, 51109095, 51179075), Natural Science Foundation of Jiangsu Province of China (Grant No. BK2009006, BK2010346, BY2011140); Special Achievements Transformation Fund in Jiangsu Province (BA2010155) and senior talents project funded Jiangsu university (12JDG044).

\section{Nomenclature}

\begin{tabular}{|c|c|c|c|}
\hline \multicolumn{4}{|c|}{ Abbreviations } \\
\hline RANS & Reynolds averaged Navier Stokes & LES & Large Eddy Simulation \\
\hline CFD & Computational Fluid Dynamics & FEM & Finite Element Method \\
\hline \multicolumn{4}{|c|}{ Notations } \\
\hline $\begin{array}{l}n_{\mathrm{s}} \\
{[\sigma]}\end{array}$ & $\begin{array}{l}\text { Safety Factor } \\
\text { Admissible Stress }\end{array}$ & $\begin{array}{l}\mathrm{b}_{2} \\
\sigma_{s}\end{array}$ & $\begin{array}{l}\text { Impeller Outlet Width } \\
\text { Stress of Yield Limit }\end{array}$ \\
\hline
\end{tabular}

\section{References}

[1] Xiao Ruofu, Wang Zhengwei, Luo Yongyao, "Dynamic Stresses in a Francis Turbine Runner Based on Fluid-Structure Interaction Analysis[J],” Tsinghua Science and Technology, 2008, 13(5): 587-592.

[2] Guerri O., Hamdouni A., Sakout A., “Fluid structure interaction of wind turbine airfoils[J],” Wind Engineering, 2008, 32(6): 539-557. 
[3] Young Y L, Motley M R, Yeung R W, “Three-Dimensional Numerical Modeling of the Transient Fluid-Structural Interaction Response of Tidal Turbines[J],” Journal of Offshore Mechanics and Arctic Engineering, 2010, 132(1): 1-12.

[4] Kato C., Yamade Y., Wang Hong, et al, "Prediction of the noise from a multi-stage centrifugal pump [C],” ASME Fluids Engineering Division Summer Meeting , 2005.

[5] Friedrich-Karl B., Hans J. D., "Comparison of Pump Impeller Orbit Curves Obtained by Measurement and FSI Simulation[C],” Proceedings of the 2007 ASME Pressure Vessels and Piping Conference, July 22-26, 2007, San Antonio, TX, United states.

[6] Wang Yang, Wang Hongyu, Xu Xiaomin, et al, "Finite element computation for impeller of stamping and welding centrifugal pump[J],” Journal of Drainage and Irrigation Machinery Engineering, 2011, 29(2): 109-113.

[7] Pei Ji, Yuan Shouqi, Yuan Jianping, "Numerical calculation for effect of fluid-structure interaction on flow field in centrifugal pump[J],” Transactions of the Chinese Society for Agricultural Machinery, 2009, 40(12): 107 112.

[8] Rikke K. B., Christian B. J., Nicholas Pedersen, "Flow in a Centrifugal Pump Impeller at Design and Off-Design ConditionsPart II: Large Eddy Simulations[J],” Journal of Fluids Engineering, 2003, 125: 73-83. 